\title{
Exact Controllability of the Heat Equation by Temporarily Strategic Actuators Borders
}

\author{
Cheikh SECK ${ }^{1,2}$ \\ ${ }^{1}$ Département de Mathématiques de la FASTEF ex ENS de l'Université Cheikh Anta Diop de Dakar, Sénégal \\ ${ }^{2}$ Laboratoire d'Analyse Numérique et d'Informatique (LANI) de l'UFR SAT, BP 234, Université Gaston Berger, \\ Saint-Louis, Sénégal \\ Correspondence: Cheikh SECK, Département de Mathématiques de la FASTEF ex ENS de l'Université Cheikh Anta \\ Diop de Dakar, Sénégal.
}

Received: September 8, 2019 Accepted: September 29, 2019 Online Published: November 13, 2019

doi:10.5539/jmr.v11n6p53 URL: https://doi.org/10.5539/jmr.v11n6p53

\begin{abstract}
The use of the strategic zone actuators, where possible, to control a system is simpler because the unknown functions depend only on the time variable. However, the restrictions and difficulties for establishing the coercivity inequality of the parabolic operator, requires looking for other methods of internal controls. So, we will generalize the concept of actuators strategic areas and deduce results of exact controllability.
\end{abstract}

Keywords: control, controllability,estimations, strategic actuator

Mathematics Subject Classification (2010): 35Q72, 93B03, 93B07, 93C20

\section{Motivations and Statement of Problem}

\subsection{Introduce the Problem}

The exact controllability of distributed systems has in recent years a net renewed interest, thanks to the development by J.L Lions (1988) Tome 1 of the HUM method (Hilbert Uniqueness Methods). It is essentially based on the uniqueness of properties suitable for the homogeneous equation by a particular choice of controls, building a Hilbert space and a continuous linear mapping of this space into its dual hilbert which is, in fact , an isomorphism which establishes the exact controllability.

For hyperbolic problems this method has yielded important results Lions (1988) Tome 2, Seck (2011), Lebeau (1994), Fursikov (1996),...; Although when the checks are supporting small Niane (1989), Jai (2002), it seems little operative, as well as for technical reasons, the multiplier method does not lead to.

As for parabolic equations any result (to our knowledge) has been prepared by this method. Also, the harmonic method is ineffective for such equations.

\subsection{Problem Statement}

This work is the logical sequence of the publications (see Seck (2019)) where a new method has been developped to solves some of these problems types. This method is based on criteria of surjectivity of a linear continuous operator and a Hilbert space in another builds directly from the exact controllability problem.

The criteria are of two types:

i. A density criterion which is a consequence of the uniqueness properties (J.L Lions (1988));

ii. A compactness criterion which follows from the parabolic nature of the operator or the regularity of control;

In both cases these criteria are easier to verify than HUM method of Lions (1988).

This method which we call exact controllability by compactness and density opens up broad prospects for the theory of the exact controllability and allows for parabolic equations of Schrödinger, plates, Navier Stokes linearized solve many issues and opening up many perspectives. 


\section{Notations and Reminders}

\subsection{Functional Analysis Reminders}

Let $\Omega$ be a regular nonempty bounded domain of $\mathbb{R}^{N}$ of border $\Gamma$, of outer unit normal $v(\sigma)$ at point $\sigma \in \Gamma$.

We denote A the unbounded operator of $L^{2}(\Omega)$ defined by

$$
\begin{array}{r}
D(A)=\left\{u \in H_{0}^{1}(\Omega) /-\Delta u \in L^{2}(\Omega)\right\} \\
A u=-\Delta u, \quad \forall u \in D(A)
\end{array}
$$

The operator A has a Hilbert base of eigenfunctions $\left(w_{k}\right)_{k \geq 1}$ such that the sequence of associated eigenvalues $\left(\lambda_{k}\right)_{k \geq 1}$ is decreasing.

Let $T>0$, we consider the following Hilberts spaces and their respective dual.

Let $\alpha \in \mathbb{R}, \beta \in\{1,0,-1\}$.

$$
F_{T}^{\alpha, \beta}=\left\{u=\sum_{k=1}^{+\infty} u_{k} w_{k} / \sum_{k=1}^{+\infty} \lambda_{k}^{\alpha} u_{k}^{2} e^{2 \lambda_{k} T \beta}<+\infty\right\}
$$

We equip $F_{T}^{\alpha, \beta}$ with the natural scalar product

$$
(x, y)_{F_{T}^{\alpha, \beta}}=\sum_{k=1}^{+\infty} u_{k} w_{k} / \sum_{k=1}^{+\infty} \lambda_{k}^{\alpha} u_{k}^{2} e^{2 \lambda_{k} T \beta}<+\infty
$$

and, the associeted norm is $\|.\|_{F_{T}^{\alpha \beta \beta}}$.

The dual of $F_{T}^{\alpha, \beta}$ is $F_{T}^{-\alpha,-\beta}$. If $x \in F_{T}^{\alpha, \beta}$ and $y \in F_{T}^{-\alpha,-\beta}$ we have:

$$
(y, x)_{F_{T}^{-\alpha,-\beta}, F_{T}^{\alpha, \beta}}=\sum_{k=1}^{+\infty} x_{k} y_{k}
$$

Remark 1 We can notice that:

i. $F_{T}^{0,0}=L^{2}(\Omega)$,

ii. $F_{T}^{1,0}=H_{0}^{1}(\Omega)$,

iii. $F_{T}^{-1,0}=H^{-1}(\Omega)$

\subsection{Case 1-D}

Let $I=] 0,1[$ an open interval of $\mathbb{R}$. We set the operator A defined by

$$
D(A)=\left\{u \in H_{0}^{1}(I) /-\frac{d^{2} u}{d x^{2}} \in L^{2}(I)\right\} ; \forall u \in D(A), A u=-\frac{d^{2} u}{d x^{2}}
$$

According to the spectral theory Brezis (1983), Hörmander (1976), A admits a Hilbert base of $L^{2}(I)$ of eigenfunctions $\left(w_{k}\right)_{k \geq 1}$ whose associated eigenvalues are $\left(\lambda_{k}\right)_{k \geq 1}$ rows in the decreasing direction where

$$
\left\{\begin{array}{ccc}
w_{k}(x) & = & \sqrt{2} \sin k \pi x \\
\lambda_{k} & = & k^{2} \pi^{2}
\end{array}\right. \text { and }
$$

See also Lebeau(1994) and Fursikov(1996).

Definition 1 An integrable square function $\mu: I \subset \Omega \longrightarrow \overline{\mathbb{R}}$ is called strategic (see El. Jai(2002,2003)) if it satisfies, for all $\phi_{0} \in L^{2}(I)$, the solution $\phi^{+}$of heat equation

$$
\left\{\begin{array}{c}
\left.\phi^{+^{\prime}}(t, x)-\Delta \phi^{+}(t, x)=0 \text { in } Q_{T}=\right] 0,+\infty[\times I \\
\left.\gamma \phi^{+}(t, x)=0 \text { in } \Sigma_{T}=\right] 0,+\infty[\times \partial I \\
\phi^{+}(0)=\phi_{0} \text { in } I
\end{array}\right.
$$


satisfies:

$$
\forall t>0, \int_{I} \mu(x) \phi^{+}(t, x) d x=0 \quad \text { then } \quad \phi_{0}=0 .
$$

\section{Remark 2}

1. In 1 dimension (see Seck (2019)), it has been proved that there are internal strategic actuators and this is the case of certain particular geometries.

2. Similarly, the Exact boundary controllability of the heat equation in 1D by strategic actuators area by the SCD Method(surjectivity by compactness and density) was done.

\section{Temporary Actuators Strategic Boundaries}

\subsection{Definition and Remark}

\section{Definition 2.}

An actuator $g(t) \in L^{2}(] 0, T[\times \Gamma)$ is said temporally strategic if

$$
\forall \phi_{0} \in H_{0}^{1}(\Omega), \text { if } \int_{\Gamma} g(t) \frac{\partial \phi^{+}}{\partial v}(t) d \sigma=0 \forall t \in \mathbb{R}_{+}^{*} \text { then } \phi_{0}=0 .
$$

\section{Remark 3.}

Due to the fact that the application $t \mapsto \int_{\Gamma} g(t) \frac{\partial \phi^{+}}{\partial v}(t) d \sigma$ is analytic then it is enough that the identity of definition is checked on an interval ]0, $T$ [ so that it is true everywhere.

\subsection{Main Result}

\section{Theorem 1.}

On any $\gamma$ open non-empty part of $\Gamma$, there exists a temporally strategic boundary operator $g(t)$ such that $\operatorname{supp}(g(t)) \subset \gamma$.

Proof. Consider $\eta \in \mathcal{D}^{+}(\Gamma)$ such that $\sup p(\eta) \subset \gamma$ and $\eta$ not identically zero.

Let $\phi_{0} \in D(A)$ such that $\forall \phi_{0 k}, \phi_{0 k} \neq 0$.

We pose $g(t)=\eta \frac{\partial \phi^{+}}{\partial_{n} u}(t)$ and let

$$
\psi_{0} \in H_{0}^{1}(\Omega) \text { such that } \int_{\Gamma} \eta \frac{\partial \psi^{+}}{\partial v} \frac{\partial \psi^{+}}{\partial v} d \sigma=0, \forall t>0
$$

so

$$
\sum_{k=1}^{+\infty} \sum_{h=1}^{+\infty} \phi_{0 k} \psi_{0 h} e^{-\left(\lambda_{k}+\lambda_{h}\right) t} \alpha_{h k}=0
$$

where

$$
\alpha_{h k}=\int_{\Gamma} \eta \frac{\partial \psi_{k}}{\partial v} \frac{\partial \psi_{h}}{\partial v} d \sigma
$$

so $\forall k \in \mathbb{N}^{*}, \forall h \in \mathbb{N}^{*}, \psi_{0 k} \alpha_{h k}=0$.

Suppose it exists $k_{0}$ such that $\forall h \in \mathbb{N}^{*}, \alpha_{h k_{0}}=0$ then $\int_{\Gamma} \eta\left(\frac{\partial w_{k_{0}}}{\partial v}\right)^{2} d \sigma=0$.

So $\frac{\partial w_{k_{0}}}{\partial v}$ on an open part of $\Gamma$ not empty but $w_{k_{0}}$ are eigenfunctions so $w_{k_{0}} \equiv 0$ in $\Omega$ which is impossible from where $\psi_{0}=0$ in $\Omega$.

\section{Exact controllability boundary of the heat equation}

Theorem 2. If $g \in L^{2}\left(\mathbb{R}_{+}^{*} \times \Gamma\right) \cap C\left(\mathbb{R}_{+}^{*}, L^{2}(\Gamma)\right)$ is temporally strategic for everything $y_{0} \in F_{T}^{-1,-1}$, it exists $\beta \in L^{2}(] 0, T[)$ such that the solution y of

$$
\left\{\begin{array}{c}
\left.y^{\prime}-\Delta y=0 \text { in }\right] 0, \infty[\times \Omega \\
\gamma y=\beta(t) g(t, \sigma) \text { in }] 0, \infty[\times \Gamma \\
y(0)=y_{0} \text { in } \Omega
\end{array}\right.
$$


verify $y(T)=0$. Proof

Formal construction of the operator $L$ ?

We multiply the previous equation (10) by $\psi^{-}(T)$ where $\psi_{0} \in F_{T}^{1,1}$ and we have

$$
\int_{\Omega} y(T) \psi^{-}(T) d x-<y_{0}, \psi_{0}>_{F_{T}^{-1,-1}, F_{T}^{1,1}}=-\int_{0}^{T} \int_{\Gamma} \beta(t) g(t, \sigma) \frac{\partial \psi^{-}}{\partial v}(t, \sigma) d \sigma d t
$$

so

$$
<y_{0}, \psi_{0}>_{F_{T}^{-1,-1}, F_{T}^{1,1}}=-\int_{0}^{T} \int_{\Gamma} \beta(t) g(t, \sigma) \frac{\partial \psi^{-}}{\partial v}(t, \sigma) d \sigma d t
$$

but if $x \in F_{T}^{-1,-1}$ and $y \in F_{T}^{1,1}$,

$$
<x, y>_{F_{T}^{-1,-1}, F_{T}^{1,1}}=\left\langle A^{-1} x^{+}(T), A x^{-}(T)\right\rangle_{L^{2}(\Omega)}
$$

from where

$$
\int_{\Omega} A^{-1} y^{+}(T) \psi^{-}(T) d x=-\int_{0}^{T} \int_{\Gamma} \beta(t) g(t, \sigma) \frac{\partial \psi^{-}}{\partial v}(t, \sigma) d \sigma d t
$$

Let $\mu$ the unique solution of the equation

$$
\left\{\begin{array}{c}
-\Delta \mu=0 \text { in } \Omega \\
\gamma \mu(t, \sigma)=g(t, \sigma) \text { in } \Gamma
\end{array}\right.
$$

We have

$$
\begin{aligned}
\int_{\Omega} A^{-1} y^{+}(T) d x & =-\int_{0}^{T} \beta(t) \int_{\Omega} g(t, \sigma) \frac{\partial \psi^{-}}{\partial v}(t, \sigma) d \sigma d t \\
& =-\int_{0}^{T} \beta(t) \int_{\Omega}\left(-\Delta \psi^{-} \mu\right) d x d t
\end{aligned}
$$

So,

$$
\begin{aligned}
<A^{-1} y^{+}(T), A \psi^{-}(T)>_{F_{T}^{-1,-1}, F_{T}^{1,1}} & =-\int_{0}^{T} \beta(t) \int_{\Omega} \psi^{\prime} \mu(t, x) d x d t \\
& =-\int_{0}^{T} \beta(t)\left\langle\Psi_{0}, \frac{\partial \mu^{-}}{\partial s}(t, t)\right\rangle_{F_{T}^{1,1} F_{T}^{-1,-1}} d t \\
& =\left\langle-\int_{0}^{T} \beta(t) \frac{\partial \mu^{-}}{\partial s}(t, t) d t, \psi_{0}\right\rangle
\end{aligned}
$$

from where

$$
A^{-1} y^{+}(T)=A^{-1}\left(-\int_{0}^{T} \beta(t) \frac{\partial \mu^{+}(T-t, t)}{\partial s}\right)^{+}(T) d t
$$

so

$$
y^{+}(T)=\int_{0}^{T} \int_{0}^{T} \beta(t) \frac{\partial \mu^{+}}{\partial s}(T-t, t) d t
$$

So, we are brought back to the first case. 


\section{References}

Brezis, H. (1983). Analyse fonctionnelle. Collection Mathématiques Appliquées pour la Maîtrise. Collection of Applied Mathematics for the Masters Degree, Thorie et Applications (Theory and Applications)(Masson, Paris, 1983).

Fursikov, A. V., \& Imanuvilov, O. Y. (1996). Controllability of evolution equations (No. 34). Seoul National University.

Hörmander, L. (1976). Linear partial differential operators. Springer Verlag, Berlin.

El Jai, A. (2003). Quelques problmes de contrôle propres aux systmes distribus. Annals of the University of CraiovaMathematics and Computer Science Series, 30, 137-153.

El Jai, A. (2002). June, Analyse régionale des systèmes distribués. ESAIM: Control, Optimisation and Calculus of Variation, 8, 663-692.

Lebeau, G., \& Robbiano, L. (1994). Contrôle exact de l'équation de la Chaleur, Prépublications Univ. Paris-Sud.

Niane, M. T. (1990). Régularité, contrôlabilité exacte et contrôlabilité spectrale de l'équation des ondes et de l'équation des plaques vibrantes, Thèse de Doctorat d'Etat, Université Cheikh Anta Diop de Dakar, Sénégal.

Niane, M. T. (1989). Contrôlabilit exacte spectrale largie des systmes distribus par action sur une partie analytique arbitraire de la frontire. Comptes rendus de l'Acadmie des sciences. Srie 1, Mathmatique, 309(6), 335-340.

Lions, J. L. (1988). Contrôlabilité exacte, perturbations et stabilisation de systèmes distribués. Tome 2, Recherches en Mathématiques Appliquées, Research in Applied Mathematics, 9, Perturbations, Masson, Paris.

Lions, J. L. (1988). Contrôlabilité exacte, perturbations et stabilisation de systèmes distribués. Tome 1,Recherches en Mathématiques Appliquées, Research in Applied Mathematics, 8, Paris.

Seck, C., Bayili, G., Sène, A., \& Niane, M. T. (2011). Contrôlabilité exacte de l'équation des ondes dans des espaces de Sobolev non réguliers pour un ouvert poygonal, Afrika Matematika.

Seck, C. (2019). Exact Boundary controllability of heat equation in $1 D$ by Strategic Actuator area by the SCD Method, Soumis.

\section{Copyrights}

Copyright for this article is retained by the author(s), with first publication rights granted to the journal.

This is an open-access article distributed under the terms and conditions of the Creative Commons Attribution license (http://creativecommons.org/licenses/by/4.0/). 